\title{
NON-CYCLIC ALGEBRAS OF DEGREE FOUR AND EXPONENT TWO WITH PURE MAXIMAL SUBFIELDS ${ }^{1}$
}

\author{
ROY DUBISCH
}

In a recent paper ${ }^{2} \mathrm{~A}$. A. Albert proved the falsity of the converse of the well known proposition that a cyclic normal division algebra contains a quantity $j$ whose minimum equation is $x^{n}=j$ in the base field of the algebra. The proof consists in giving an example of a noncyclic normal division algebra containing a quantity $j$ as described above. The algebra described in that example was of degree and exponent four. It is the purpose of this paper to show that the exponent does not affect the property, and this we shall do by constructing similarly an algebra of degree four whose exponent is two.

We shall actually prove the following theorem.

TheOREM. Let $\xi$ and $\eta$ be independent indeterminants over the field $R$ of real numbers, $K=R(\xi, \eta)$. Then there exist non-cyclic normal division algebras of degree four and exponent two over $K$ such that $t^{4}=\gamma$ in $K$, $t^{2}$ not in $K$, for some quantity $t$ in each algebra $A$.

To make our proof we use the known ${ }^{3}$ property stating that a normal division algebra of degree four has exponent two if and only if it is expressible as a direct product of two algebras of degree two. Therefore we may take our desired algebra $A$ to be

$$
\begin{array}{ll}
A=B \times C=(1, i, j, i j) \times(1, x, y, x y), \\
j i=-i j, \quad i^{2}=u, \quad j^{2}=a, \quad u \neq 0, a \neq 0 \text { in } K, \\
y x=-x y, \quad x^{2}=v, \quad y^{2}=b, \quad v \neq 0, b \neq 0 \text { in } K .
\end{array}
$$

We seek first a quantity $t$ with minimum equation $t^{4}=\gamma$ in $K$. Now if we take $t=a_{1} i+a_{2} j+a_{3} i j$, where $a_{1}, a_{2}, a_{3}$ are in $K(x)$, and if we put

$$
a_{1}=c_{1}+c_{2} x, \quad a_{2}=d_{1}+d_{2} x, \quad a_{3}=f_{1}+f_{2} x, \quad c_{i}, d_{i}, f_{i} \text { in } K,
$$

we can easily compute that if

$$
a=-\frac{c_{1}^{2} u+\underset{c_{2}}{2} u v}{d_{1}^{2}+d_{2}^{2} v-f_{1}^{2} u-f_{2}^{2} u v},
$$

\footnotetext{
${ }^{1}$ Presented to the Society, April 13, 1940.

2 A. A. Albert, Non-cyclic algebras with pure maximal subfields, this Bulletin, vol. 44 (1938), pp. 576-579.

${ }^{3}$ A. A. Albert, Normal division algebras of degree four, Transactions of this Society, vol. 34 (1932), p. 369.
} 
and if

$$
b_{2}=2 c_{1} c_{2} u+2 d_{1} d_{2} a-2 f_{1} f_{2} u a \neq 0,
$$

the algebra $A$ defined by (1) contains a quantity $t$ such that $t^{4}=\gamma$ in $K$, $t^{2}$ not in $K$.

In proving $A$ a non-cyclic division algebra we use the following method. ${ }^{4}$ Let $z^{2}=\delta^{2}+\epsilon^{2}=\Delta$ in $K, \delta$ and $\epsilon$ in $K$ with $L=K(z)$ a quadratic field over $K$. The algebras $B_{0}=B \times L$ and $C_{0}=C \times L$ over $L$ are generalized quaternion algebras over their reference field $L$. Furthermore, $A_{0}=A \times L$ is the direct product $A_{0}=B_{0} \times C_{0}$. Then it is known ${ }^{5}$ that a necessary and sufficient condition that $A_{0}$ over $L$ shall be a division algebra is that the quadratic form

$$
Q \equiv u \lambda_{1}^{2}+a \lambda_{2}^{2}-u a \lambda_{3}^{2}-\left(v \lambda_{4}^{2}+b \lambda_{5}^{2}-v b \lambda_{6}^{2}\right)
$$

in the variables $\lambda_{1}, \lambda_{2}, \cdots, \lambda_{6}$ in $L$ shall not vanish for any $\lambda_{1}, \cdots, \lambda_{6}$ not all zero in $L$.

If $J=R[\xi, \eta]$ is the integral domain of all polynomials in $\xi$ and $\eta$ with real coefficients, it is obvious that we may, without loss of generality, take the $\lambda_{i}$ in (5) to be in $J$. Hence we may write

$$
\lambda_{i}=\alpha_{i}+\beta_{i} z, \quad i=1, \cdots, 6,
$$

where the $\alpha_{i}$ and $\beta_{i}$ are in $J$. Then $\lambda_{i}^{2}=\left(\alpha_{i}^{2}+\beta_{i}^{2} \Delta\right)+2 \alpha_{i} \beta_{i} z$ so that if

$$
P_{i}=\alpha_{i}^{2}+\beta_{i}^{2}, \quad Q_{i}=2 \alpha_{i} \beta_{i},
$$

the equation $Q \equiv 0$ becomes

$$
\begin{aligned}
& u P_{1}+a P_{2}-u a P_{3}-v P_{4}-b P_{5}+v b P_{6} \\
& \quad+\left(u Q_{1}+a Q_{2}-u a Q_{3}-v Q_{4}-b Q_{5}+v b Q_{6}\right) z \equiv 0 .
\end{aligned}
$$

But 1 and $z$ are linearly independent ${ }^{6}$ with respect to $K$ so that (8) implies that

(9) $\phi(\xi, \eta) \equiv u P_{1}+a P_{2}-u a P_{3}-v P_{4}-b P_{5}-v b P_{6} \equiv 0$.

Now if in (3) we let $c_{1}=c_{2}=d_{1}=1, d_{2}=f_{1}=f_{2}=0$, we obtain

$$
a=-(u+u v) \text {. }
$$

${ }^{4}$ This is the device used by A. A. Albert in his paper $A$ construction of non-cyclic normal division algebras, this Bulletin, vol. 38 (1932), pp. 452-453.

5 A. A. Albert, On the Wedderburn norm condition for cyclic algebras, this Bulletin, vol. 37 (1931), p. 311, Theorem 3 .

${ }^{6}$ It is obvious that we can have this condition hold by a suitable choice of $\delta$ and $\epsilon$. 
Note that these choices also satisfy (4). Substituting this value for $a$ in (3) we have

(11) $\phi(\xi, \eta)=u\left(P_{1}-P_{2}\right)-u v P_{2}+u^{2} P_{3}+u^{2} v P_{3}-v P_{4}-b P_{5}+v b P_{6}$.

With these choices for the parameters of our algebra $A$ is easy to prove by the well known method of an argument ${ }^{7}$ on the degrees of the polynomial in $(7)$ that $\phi(\xi, \eta)$ cannot be identically zero unless all of the coefficients are zero. From this it follows that $A_{0}$ over $L$ is a division algebra over $L$; so is algebra $A$ a division algebra over $K$. By its form it is a normal division algebra of degree four and exponent two over $K$.

The proof of the non-cyclic character of $A$ is exactly the same as that given by A. A. Albert in a previous paper, ${ }^{8}$ and with this we have the desired theorem.

Wilson Junior COLlege

${ }^{7}$ A similar argument was employed by A. A. Albert in the paper last cited.

8 This Bulletin, vol. 38 (1932), p. 454. 